\title{
Vers une pensée migrante
}

\section{Severino Ngoenha et Laura Ferilli}

Cet article prétend dépasser la conception réduite de l'interculturalité en tant que simple outil de gestion de l'immigration telle qu'elle est souvent théorisée et surtout pratiquée. Dialectiquement, au lieu de disputer le lien entre migration et interculture, nous le renforçons en proposant une pensée migrante. Celle-ci consiste en une prise de conscience de la pan-migratio: c'est-à-dire que nous vivons dans un monde de voyage perpétuel, non seulement des personnes mais aussi des marchandises, des idées, des pensées et des représentations. Ainsi, la migratio ne saurait se réduire aux migrations économiques. Prendre au sérieux cette dimension constitutive de notre époque signifie prendre au sérieux les différents niveaux d'interculture que nous vivons: la constant dialogue intrasuisse entre les cultures et les langues différentes, la question de la réconciliation intraeuropéenne et surtout, l'interculturation du monde.

\section{Introduction}

L'association entre interculturalité et migration est devenue si communément admise qu'elle a fini par masquer les dimensions économiques, politiques, géographiques, générationnelles ou de genres - que les approches interculturelles devraient comprendre - et réduit ainsi toute la complexité de ces dernières à l'immigration.

A partir de ce constat, quelles ouvertures théoriques pouvons-nous envisager pour sortir du piège dans lequel l'interculturalité s'est laissé enliser? Dans cet article, nous prendrons le risque épistémologique de proposer une alternative à l'impasse herméneutique créée par le lien entre interculturalité et migration. Dialectiquement, nous tenterons de renforcer ce lien plutôt que de le disputer.

Pour ce faire, notre position sera de prendre la migratio (déplacement d'un lieu à un autre) pour ce qu'elle est, c'est-à-dire non comme un épiphénomène de la société contemporaine - nommée aussi société de l'information et du savoir (Berthoud, Ischy \& Simonis, 2002) -, mais comme constitutive de cette dernière. 
Il est vrai que les migrations visibilisées sont principalement constituées par des personnes. Mais ne sont-elles pas moins importantes - par la densité et la durée - que les migrations des biens et services économiques? Et que dire de la circulation exponentielle de l'information - principal critère d'identification de notre société - qui ne connaît ni passeport ni douane?

Même si nous arrivions, par des mesures politiques, à maîtriser les migrations des individus, la circulation des biens n'en serait pas entachée pour autant car la déconnexion des économies, suggérée par Samir Amin dans les années 80, est improbable. A fortiori, même dans un tel scénario, il est difficile d'imaginer que la circulation des images et des messages - qui contiennent des valeurs et contrevaleurs, des croyances, des représentations et des idéaux - véhiculés par les technologies, arrêtera sa progression et le processus de migratio.

En effet, nos chaînes de télévision et les blogs de toute sorte continueront à nous présenter les conflits au Moyen-Orient et au Darfour, la pauvreté en Afrique, la progression économique de l'Inde et de la Chine ainsi que la nécessité écologique de la mobilisation de tous pour sauver la planète.

A partir de ce que nous venons d'exposer, il nous semble problématique de réduire, d'une part, la migratio aux immigrations économiques des populations et, d'autre part, l'interculturalité à celles-ci car, même sans déplacement des personnes, par le biais de la circulation des biens et de l'information, il y aurait, inexorablement, «interculturation du monde» (Demorgon, 2000).

Dès lors, nous voyons se dessiner l'exigence d'une nouvelle forme de pensée qui tienne compte de la dimension essentielle de la migratio dans notre "village global» et qui reconnaisse le visage humain de tous les villageois ainsi que leur manière spécifique de se signifier à eux-mêmes et aux Autres (ce que nous appelons la culture): il s'agit de la pensée migrante, posée à la source de l'interculturalité.

Léducation, dont la mission est de préparer les jeunes au monde dans lequel ils vont vivre, ne peut faire l'économie de la pensée migrante sans se trahir.

\section{Complexifier l'interculturalité}

\section{Interculture et immigration}

La tendance est, tant au niveau de la recherche, de la politique que des pratiques, de réduire systématiquement l'interculturalité au champ de l'immigration «qui pose problème». C'est dans cette logique que nous la retrouvons généralement intégrée à la sociologie des migrations en France et à la pédagogie destinée aux enfants migrants en Italie.

La simplification de l'interculturalité ne semble pas avoir épargné la Suisse. À titre d'exemple, le PNR 39, projet du Fonds national suisse de la recherche scientifique s'étendant de 1995 à 2002, "Migrations et relations interculturelles», 
poursuivait le but de «mieux comprendre les phénomènes migratoires et les relations interculturelles dans leur complexité». Pour ce faire,

un budget de huit millions fut alloué à 28 travaux de recherche dans les domaines suivants: santé, affaires sociales, travail, formation, marché de l'emploi, développement urbain, droit, histoire et politique. Les connaissances acquises par le PNR 39 ont permis d'influencer directement les usages relatifs aux migrations, par exemple les modèles d'intégration urbaine de Bâle et de Berne, ainsi que l'élaboration de la nouvelle loi sur les étrangers ou encore l'ouverture de forums axés sur les recherches migratoires à Neuchâtel et, récemment, à Varsovie. ${ }^{l}$

Dans la suite du texte qui présente les résultats de ces recherches, on peut lire: "Alors que les 'anciens' migrants d'Europe du Sud se sont relativement bien établis comme travailleurs immigrés, les 'nouveaux' migrants, de l'ex-Yougoslavie, d'Asie ou d'Afrique par exemple, sont beaucoup plus marginalisés. Comme l'atteste un projet récemment achevé du PNR 39, toutes les catégories d'immigrants subissent une discrimination salariale systématique sur le marché de l'emploi, qui découle directement de leur statut de séjour (type de permis). Après déduction de paramètres variables comme la formation ou l'expérience, les différences salariales par rapport aux Suisses demeurent encore de 3,6\% chez les titulaires d'un permis d'établissement, de 4,5\% chez les résidants à l'année, de $15 \%$ chez les anciens saisonniers et, comme l'indique une autre enquête, de 45\% chez les requérants d'asile. Les scientifiques constatent aussi que les demandeurs d'asile devenus depuis indispensables dans plusieurs secteurs de l'économie - prennent toujours plus le rôle des anciens travailleurs immigrés et, comme ces derniers à l'époque, servent de «tampon conjoncturel». ${ }^{2}$

Le réductionnisme des approches interculturelles apparaît de façon encore plus patente dans le domaine de l'éducation où celles-ci semblent (con)cerner non seulement l'immigration, mais plus spécifiquement la migration des enfants a priori pauvres et faibles.

À ce propos, la rubrique «Langue et éducation interculturelle» du site internet de la Conférence suisse des directeurs cantonaux de l'instruction publique (CDIP) est très éloquente. En effet, les publications que nous y trouvons, outre le rapport concernant la formation des enseignantes et enseignants aux approches interculturelles, concernent toujours les élèves issus d'une migration perçue implicitement comme défavorisée: "Scolarisation des enfants portugais en Suisse», "Des élèves italiennes et italiens en Suisse», "Le parcours scolaire et de formation des élèves immigrés à 'faibles' performances scolaires», etc.

De même, pour le projet vaudois du Département de la formation et de la jeunesse «Bus plurilingue-Livres migrateurs» qui se déroula d'octobre 2004 à janvier 2005 et dont le but était de proposer aux élèves des cycles primaires «des livres écrits dans diverses langues pour les rendre attentifs à la richesse linguistique et culturelle du milieu dans lequel ils vivent» ${ }^{3}$, a-t-il été nécessaire de préciser que ce projet n'était pas destiné uniquement aux élèves allophones et pouvait intéres- 
ser toutes les classes et que, par conséquent, «tous les enseignant-e-s de la région (et non seulement ceux du CIF (cours intensifs de français) ou des classes d'accueil) sont invités à participer à cette action jusqu'à fin janvier 2005». ${ }^{4}$ D'après le rapport d'évaluation de ce projet, malgré l'invitation élargie de la Direction pédagogique, le but n'a pas été atteint car la composition de l'échantillon d'enseignantes et d'enseignants présents lors des manifestations organisées autour du «Bus plurilingue» montre que «les classes touchées sont des classes plutôt hétérogènes avec de fortes proportions d'allophones» (Batori, 2005, p. 9).

Peut-être le réductionnisme dans lequel se complaît le concept d'interculturalité en pédagogie helvétique a-t-il trouvé un nid dans le cadre théorique au sein duquel les recherches en éducation se sont installées. En effet, la philosophe Martine Abdallah-Pretceille, qui représente une des références théoriques principales pour l'interculturalité dans l'enseignement et la recherche, est fréquemment citée dans les projets de l'instruction scolaire en Suisse romande tout comme dans les rapports de la CDIP susmentionnés.

En substance, dans sa théorie exposée en 1986, Martine Abdallah-Pretceille situe la genèse de l'interculturalité en 1975 dans le milieu scolaire français, en lien avec la décolonisation et les problèmes - allant des échecs scolaires des enfants aux difficultés du corps enseignant - entraînés par la présence toujours plus importante d'enfants provenant des anciennes colonies sur le sol français métropolitain.

Le mérite de cette approche est d'avoir pris au sérieux la situation de l'époque - c'est-à-dire une certaine urgence face aux difficultés et souffrances des élèves, des enseignants et des parents - et élaboré des propositions concrètes pour les surmonter. On peut également saluer le fait que la philosophe française ait situé l'interculturalité au cœur de l'instruction publique, ce qui a pour effet de lui conférer une position et un rôle particuliers dans la société car, ce faisant, Abdallah-Pretceille présuppose que l'école remplit une mission bien particulière, différente des autres institutions étatiques en charge du bien-être public - telle que la police par exemple. ${ }^{5}$ L'école revêt la fonction de transmettre un vocabulaire et une grammaire des valeurs communs à un ensemble qui, a priori, pourrait être appréhendé comme hétérogène. Ainsi, cette approche permet d'attribuer à l'école un rôle majeur dans la construction du monde de demain et met en exergue toute la noblesse du métier d'enseignante et d'enseignant, pas en tant que transmetteur de connaissances spécifiques - telles que les mathématiques, la géographie, l'histoire ou l'allemand -, mais en tant que «forgeronnes et forgerons» des consciences citoyennes chez les enfants - qu'ils soient indigènes ou qu'ils proviennent d'ailleurs - afin qu'ils puissent partager des repères communs et coopérer.

Néanmoins, cette acception de la perspective interculturelle est réductrice et le piège dans lequel elle a fait tomber le concept d'interculture est que l'objet prioritaire et presque unique de la pédagogie interculturelle soit devenu, et demeure, malgré les différents masques qu'elle revêt, les enfants issus de la migra- 
tion et identifiés principalement par leurs manques (pédagogie compensatoire). Par conséquent, elle empêche d'appréhender la complexité des multiples situations scolaires contemporaines qui, dépassant de loin l'unique problématique de l'immigration des personnes, concerne chaque actrice et acteur de la société en contact avec l'omniprésence de la migratio des individus, il est vrai, mais surtout des choses, des idées et des représentations.

\section{Interculturalité comme réconciliation intraeuropéenne}

Le philosophe et sociologue Jacques Demorgon pose la genèse de l'interculturel dans le processus de réconciliation intraeuropéen qui aurait démarré en 1963 avec la signature de l'Accord portant sur la création de l'Office franco-allemand pour la Jeunesse (découlant du Traité de coopération franco-allemande de la même année). ${ }^{6}$ Selon l'article 2 de l'Accord,

l'Office a pour objet de resserrer les liens qui unissent les jeunes des deux pays, de renforcer leur compréhension mutuelle et, à cet effet, de provoquer, d'encourager et, le cas échéant, de réaliser des rencontres et des échanges de jeunes».7

Ainsi furent constitués des cycles de rencontres internationales animés par une équipe pluridisciplinaire d'enseignants universitaires et de chercheurs professionnels appartenant aux deux pays.

L'une des règles de ces rencontres "expérimentales» était l'acceptation des monolingues et la non-présence d'interprètes professionnels, le groupe devant trouver en son sein les ressources linguistiques nécessaires. [...] Les conditions étaient réunies pour permettre aux participants et aux chercheurs d'aborder en profondeur nombre des problématiques interculturelles franco-allemandes et autres (Demorgon, 2002, p. 5).

Contrairement à Abdallah-Pretceille, Demorgon «cherche le chemin de l'invention de l'interculturel»(Hess, in Demorgon, 2002, p. 4), non en 1975 mais plus de dix ans auparavant, ce qui n'est pas sans conséquences dans la conception des approches interculturelles. En effet, situées à cette époque, elles ne peuvent plus trouver leur source dans le processus de décolonisation et d'immigration des anciennes colonies françaises.

La conséquence logique de cette perspective est celle de libérer l'interculturel des conflits entre deux cultures conçues comme radicalement différentes et surtout axiologiquement inégales. Il s'agit, au contraire, d'une conception de l'interculturel en tant que dialogue entre les héritiers de Goethe et Baudelaire, Renoir et Dürer ou encore Beethoven et Berlioz, c'est-à-dire du dialogue entre deux nations qui se reconnaissent de cultures d'égale dignité.

En définitive, en proposant la réconciliation intraeuropéenne comme point de départ de l'interculturel, la position de Demorgon peut permettre de dépasser la dichotomie entre autochtones et étrangers, demandeurs et donneurs, aussi bien que la hiérarchisation implicite des cultures que cela suppose, dans laquelle a été enfermée l'interculturalité. En effet, il ne se traite ici nullement d'immigration et des problèmes posés par celle-ci aux nations d'accueil mais bien d'un pro- 
cessus de réconciliation - entamé il y a quarante-cinq ans - entre le peuple allemand et le peuple français, dans le but de mettre fin aux conflits nationalistes en Europe.

\section{Interculturation du monde}

Deux ans plus tard, le même Demorgon dépasse le cadre de la réconciliation intraeuropéenne et parle de «l'interculturation du monde» (2000). Pour défendre cette thèse, il divise l'histoire humaine en quatre grandes périodes d'organisation sociale post-préhistoriques dans lesquelles l'activité sociétale se différencie en quatre grands secteurs: religieux, politique, économique et informationnel. Dans la première forme de société, communautaire-tribale, aussi bien que dans celle qui lui succède, celle des royaumes et empires, le religieux et le politique organisent la société en contrôlant l'économie et l'information. Les nations marchandes, forme sociétale qui suit, ont émergé grâce au renversement de la hiérarchie précédente: «Une nation marchande repose en effet sur l'association des acteurs économiques et informationnels qui contrôlent le religieux et le politique» (Demorgon, 2005, p. 199). La quatrième forme, dans laquelle nous nous trouverions aujourd'hui, est informationnelle mondiale, caractérisée à la fois par l'augmentation quantitative de l'information et l'accélération exponentielle de sa circulation.

Par conséquent, selon le philosophe français, nous assistons, de manière irréversible et structurel, à un double processus: à l'explicite et controversée globalisation économique et à une implicite mais non moins insidieuse mondialisation culturelle. ${ }^{8}$ Avec les échanges et les collaborations économiques contrôlées circuleraient des éléments culturels moins perceptibles mais tout aussi conséquents. Cette approche est également défendue par d'autres penseurs, tels le philosophe Gérald Leclerc dans La mondialisation culturelle (2000) et le politologue Paul Ariès dans La McDonaldisation du monde (1998).

Pour illustrer le propos de Demorgon par un exemple qui nous est proche, le drame quasi national qu'a entraîné en 2000 le rachat d'Alusuisse - patrimoine helvétique, tout comme le fut Swissair - par Alcan (dont le siège est au Canada), a montré qu'au-delà d'une simple transaction financière, s'est joué le risque d'une transaction culturelle, ou, pour le dire avec les mots de Daniel EtoungaManguelle (1991), d'un «ajustement culturel». Si, à la suite d'une profonde crise, des arrangements ont pu être trouvés entre les employés d'Alusuisse et Alcan, afin de ne pas trop modifier la culture de l'entreprise, il n'est pas certain qu'il en sera de même aujourd'hui. En effet, la multinationale américaine Alcoa, numéro un mondial de l'alumium, a lancé en mai de cette année une OPA hostile de 33 milliards de dollars pour racheter Alcan, numéro deux mondial. Lorsque Alcoa a racheté les deux plus grandes usines extractrices d'aluminium en ex-URSS dans les années 90, cela n'a pas été - et de loin - qu’une opération financière. Les méthodes de travail ont changé, la gestion des ressources naturelles s'est rationalisée, des connaissances humaines ont été dilapidées par la restructuration décidée aux 
Etats-Unis, les cadres ont dû se mettre à l'anglais, etc. La culture managériale américaine s'est imposée à la population concernée par ce rachat. Ainsi, il est légitime de nous demander si, dans le cas où l'OPA aboutissait, les personnes touchées de près et de loin par Alcoa en Suisse ne devront pas subir un «ajustement culturel» allant de pair avec un «ajustement économique» les dépassant largement.

\section{L'interculturalité intrahelvétique}

Préambule:

Le peuple et les cantons suisses, [...] déterminés à vivre ensemble leurs diversités dans le respect de l'autre et l'équité, [...].

Art. 2 al. 2

Elle [la Constitution] favorise la prospérité commune, le développement durable, la cohésion interne et la diversité culturelle du pays. Art. 69 al. 3

Dans l'accomplissement de ses tâches, elle tient compte de la diversité culturelle et linguistique du pays.

Art. 70 al. 2

Les cantons déterminent leurs langues officielles. Afin de préserver l'harmonie entre les communautés linguistiques, ils veillent à la répartition territoriale traditionnelle des langues et prennent en considération les minorités linguistiques autochtones.

Le préambule de la Constitution tout comme les articles susmentionnés peuvent être interprétés comme un plaidoyer en faveur de l'interculturalité intraSuisse. En effet, y sont mentionnés la volonté de faire rimer la cohésion interne avec la diversité linguistico-culturelle (art. 2 et 69), le respect de l'autre et l'équité, ainsi que la détermination de "préserver l'harmonie entre les communautés linguistiques (art. 70). De plus, l'article 2 conçoit la Constitution comme un moyen de favoriser la "prospérité commune».

Pourtant, bien que la diversité intrahelvétique - revendiquée et défendue déjà dans l'Acte de Médiation par Napoléon en 1803 - et l'entente interculturelle soient devenues, à côté de la démocratie directe, une sorte de référence, de marqueur d'une identité plurinationale en opposition aux grandes patries mononationales voisines que sont la France, l'Allemagne et l'Italie (Widmer, 2005), cet idéal de cohabitation n'est pas des plus faciles et demande des efforts de tous les groupes culturels et linguistiques au quotidien.

À titre d'exemple, la question de la «bonne» politique des langues dans le système éducatif a toujours eu de la difficulté à trouver un consensus entre tous les acteurs. Bien que l'instruction relève de la compétence des cantons, certains domaines, comme l'enseignement des langues, doivent faire l'objet d'accords intercantonaux. Ainsi en est-il de l'introduction de l'apprentissage dans les classes primaires d'une langue seconde, nationale, préconisée déjà dans les recommandations de la CDIP en 1975. 
Par ailleurs, à la fin des années 90, les directeurs cantonaux ont passé un accord prévoyant l'enseignement de deux langues «étrangères» au primaire, sousentendus l'anglais et une seconde langue nationale, ce qui démontre une volonté de renforcer la cohésion nationale sans pour autant prétériter ce que Demorgon appelle l'interculturation du monde.

Et pourtant, nonobstant ces efforts pour «donner un ordre politique à la diversité linguistique en Suisse» (Widmer, 2005, p. 3) et parallèlement se mettre en phase avec la globalisation et son symbiote qu'est l'anglicisation ${ }^{9}$, plusieurs cantons suisses alémaniques ont successivement vu, depuis 2004, des initiatives populaires remettre en cause ces pactes intercantonaux en proposant de n'enseigner que l'anglais dans les classes primaires, donnant la primauté à l'interculturation du monde au détriment de l'interculturalité intra-helvétique. Ceci eut pour effet de soulever des interrogations quant à l'actualité du modèle suisse. En effet, en 2005, le sociolinguiste et historien Sandro Bianconi parla de "crise aiguë actuelle du plurilinguisme en Suisse» (p. 78) et d'une «époque où la solidarité confédérale n'est plus une valeur appréciée, où l'on assiste au triomphe du pragmatisme utilitariste centré sur l'anglais» (p. 81). Heureusement, la volonté de vivre ensemble dans le respect des différences fut réaffirmée par les votations populaires, via l'option pour l'enseignement de deux langues «étrangères» à l'école primaire, ce qui eut pour conséquence de renforcer le pacte confédéral interculturel, y compris dans le domaine linguistique. Malgré cette difficulté de parcours dans l'exercice interculturel que la Suisse a traversée, la volonté de la convivence dans l'unité et dans la diversité - à travers la vox populi - l'a remporté sur les velléités régionales ethnocentrées qui nous guettent et nous rappellent la nécessité d'une vigilance interculturelle permanente.

Malgré son issue positive, cette crispation autour de l'enseignement des langues nationales démontre la fragilité de l'entente confédérale au quotidien - pensons au nombre de fois où l'épouvantail du Röstigraben est mobilisé - et permet de mettre en lumière l'importance du dialogue entre les diverses cultures la composant. L'interculture, dans l'exemple ci-dessus, n'a rien à voir avec la présence des élèves immigrants, mais avec la convivence intrahelvétique, d'une part, et le processus de globalisation d'autre part.

Toutefois, la controverse autour des langues n'est pas l'unique pierre d'angle sur laquelle nous pouvons poser notre thèse de l'interculturel comme levier d'un continuel dialogue intrahélvétique. De la vaste recherche dirigée par Uli Windisch (1992) sur les relations quotidiennes entre les Suisses romands et les alémaniques ressort clairement à quel point l'entente confédérale repose sur une négociation permanente, jamais acquise, encore à construire. $S$ 'attardant sur Fribourg, il met en exergue le fait que la coexistence harmonieuse est périodiquement rongée par des revendications alémaniques et des crispations romandes. Au-delà du principe de territorialité des langues et des frontières linguistiques éléments les plus palpables peut-être -, grouillent des conflits liés tour à tour à 
l'évolution déséquilibrée de l'économie fribourgeoise et de son industrialisation, à des oppositions entre anciens et nouveaux habitants et entre ruraux et citadins. Toutes ces sources de tension en terre fribourgeoise - mais également présentes ailleurs - indiquent autant de dimensions susceptibles de mobiliser les approches interculturelles.

A celles-ci, nous pourrions très certainement ajouter celle du décalage des références axiologiques entre les générations, ce qui n'est pas propre à la Suisse. Cela a, par ailleurs, amené le philosophe français Alain Finkielkraut à décréter, dans La défaite de la pensée (1988), que le drame de l'école - mais peut-être le constat s'étend-il à l'entier de notre société - est que les enseignantes et enseignants sont modernes alors que les élèves sont postmodernes.

\section{Les approches interculturelles en éducation en Suisse}

Cristina Allemann-Ghionda, Claire de Goumoëns et Christiane Perregaux ont publié, en 1999, un rapport concernant l'enseignement des approches interculturelles dans la formation des maîtres en Suisse, dont les conclusions ont été reprises et mutées par la CDIP en recommandations, à l'intention des Hautes écoles pédagogiques naissantes, quant à ses exigences pour la formation des enseignantes et enseignants.

Il est vrai que la mention d'une formation aux approches interculturelles, couplée à la nécessité, pour les instances de politiques scolaires, de prendre des mesures afin de gérer l'hétérogénéité croissante des classes, apparaît déjà dans les recommandations de 1972. Celles-ci, à l'instar des travaux d'Abdallah-Pretceille, pointent exclusivement les enfants migrants ou, plus précisément, contiennent des consignes quant à la manière d'être des enseignantes et des enseignants vis-àvis de ces enfants "particuliers». Celles de 2000, en revanche, visent, à travers la formation des maîtresses et des maîtres, une pédagogie qui se veut interculturelle, c'est-à-dire ouverte à tous les élèves et comprenant toutes les disciplines. En effet, d'une part, les auteures reprennent à leur compte, dès le premier paragraphe, le lien étroit, presque intrinsèque, entre migration et interculturalité en écrivant que «la complexité de l'enseignement dans des classes hétérogènes et le dénuement ressenti par les enseignantes et enseignants face à l'étrangeté de l'autre, sa reconnaissance, son rapport aux savoirs, sa distance linguistique et culturelle, son écart à la norme entraînent fréquemment la marginalisation d'élèves issus de familles migrantes» (p. 7), et d'autre part, elles portent aussi l'accent sur l'éducation interculturelle adressée à tous les élèves.

Paradoxalement, malgré l'élargissement évident de la conception de l'interculturalité, les deux axes proposés, «intégration des élèves issus de la migration» et "éducation interculturelle adressée à tous les élèves», sont maintenus distincts l'un de l'autre car, selon les auteures, ceux-ci ne feraient pas «immédiatement appel aux mêmes enjeux» (p. 10).

Cette distinction entre la catégorie «élèves migrants» et la catégorie «tous les élèves» nous semble problématique dans la mesure où elle ne tient pas compte de 
la dimension plurielle de la citoyenneté - communale, cantonale, suisse, européenne, mondiale - et donc de la nécessité de doter les futur-e-s citoyen-ne-s des outils à la fois axiologiques et de savoir-faire leur permettant d'intégrer - d'une manière responsable et harmonieuse - les différents niveaux de leurs appartenances culturelles au sein du village global dans lequel ils vivent déjà et qui se restreint chaque jour davantage de par les multiples migratio.

On peut donc, à l'instar de Jacques Demorgon et son «interculturation du monde» (2000) ou Gérald Leclerc et sa «mondialisation culturelle» (2000), affirmer que c'est le monde entier et tous ses éléments qui, virtuellement ou concrètement, migrent. Ainsi, la distinction entre élèves migrants et non migrants perd sa substance puisque nous sommes toutes et tous des personnes migrantes - en déplacement réel ou virtuel - et dès lors, les enjeux de l'interculturalité à l'école concernent véritablement l'ensemble de la population. Ainsi, comme nous l'avons déjà mentionné plus haut, la relation entre migratio et interculturalité non seulement se trouve renforcée dans notre perspective, mais découle de leur consubstantialité dans l'émergence de cette forme de pensée fertilisante qu'est la pensée migrante.

\section{Pensée migrante, voyage et école}

\section{Du voyage "Gefahr» au voyage "Erfahrung»}

Migratio et interculturalité sont deux notions consubstantielles à la notion du voyage. Les études linguistiques ont démontré que du mot Fahrt-voyage en allemand - dérivent les mots Erfahrung - expérience - et Gefahr - danger -, deux concepts qui pourraient bien symboliser les deux principales positions - une plus constante et l'autre plus marginale - qui ont toujours caractérisé l'Occident face à l'Autre.

La première met l'accent sur la dimension positive de toute rencontre, vue véritablement comme porteuse de richesses humaines. C'est dans cette ligne que s'inscrivent les pensées d'Hérodote, Montaigne, Rousseau ou encore Montesquieu, et dont l'apogée se trouve dans l'anthropologie savante telle qu'elle émerge à partir du XIXe siècle.

La seconde position insiste sur le danger que peut représenter, pour l'ordre culturel interne (crainte de Platon ou du Descartes des Méditations métaphysiques), toute rencontre avec l'altérité. À ce titre, il importe soit de limiter les contacts avec elle, soit alors de l'assimiler au même. C'est dans cette perspective qu'il faut comprendre le voyage missionnaire, tout comme le voyage philanthropique et colonial.

Un des dangers qui guette les approches interculturelles comme elles sont conçues, mais surtout pratiquées actuellement, est celui d'emboîter le pas au voyage 
assimilationniste, propre aux missionnaires, à partir du postulat selon lequel, étant donné que les païens, les sauvages, les barbares sont désormais intra-muros, il s'agirait de former des spécialistes - missionnaires des temps modernes - chargés de leur conversion culturelle.

Une alternative à ce danger serait de recentrer l'interculturel autour du voyage anthropologique qui postule, si ce n'est l'égalité des différences culturelles, au moins leur totale respectabilité et la nécessité de leur existence pour l'humanité.

Il ne faut pas oublier que l'hétérogénéité, même si elle est beaucoup plus dérangeante, même si elle est beaucoup plus difficile à penser, est source de vie. C'est avec le "contradictoriel» (S. Lupasco, G. Durand) que commence l'existence, alors que l'identique ou l'homogène bien que plus pacifique ou plus cernable reste potentiellement mortifere (Maffesoli, 2003, p. 6).

En effet, parce qu'il est et qu'il demeure différent, l'Autre suscite l'intérêt.

Dans ce sens-là, le défi de la pensée migrante est de dissocier le voyage du danger, c'est-à-dire de la crainte de l'Autre et ses corollaires que sont soit sa mise à distance, soit son assimilation. Ceci aurait pour effet de laisser le champ libre au voyage en tant que Erfahrung, expérience de l'Autre qui nous amène à notre propre enrichissement anthropologique et culturel.

L'école, par sa position charnière entre la prime enfance et l'âge adulte, le local et le global, le passé et le futur, peut jouer un rôle déterminant dans la construction d'un monde migrant fécond, où l'Erfahrung du voyage anthropologique l'emporterait enfin sur la réduction au même du voyage missionnaire.

\section{Propositions pour la formation à la pensée migrante et pour la recherche}

Les cours d'interculturalité, en adéquation avec la pensée migrante, destinés aux futur-e-s enseignantes et enseignants, devraient s'orienter vers deux axes de réflexion.

Le premier consisterait à réfléchir à cette particularité de notre époque : nous sommes dans «un monde en voyage». Le voyage omniprésent "de tout» est constitutif de notre quotidien. Cette prise de conscience a partiellement eu lieu au niveau économique. À travers les mouvements boursiers, les marchés mondialisés, les délocalisations d'entreprises, etc., la dimension économique de la pensée migrante est la plus directe à saisir. Il faut dire que son attirail technologique et communicationnel de plus en plus répandu dans les chaumières y a largement contribué. Toutefois, il s'agit bien de rendre compte que ce ne sont pas que les capitaux transitant entre les Bourses de New York, Paris et Tokyo qui constituent la pensée migrante et la nécessité d'une approche interculturelle. Au contraire même, ce sont tous les autres éléments voyageurs qui méritent notre attention dans la mesure où nous sommes plus directement et plus concrètement aux pri- 
ses avec eux dans notre quotidienneté. Parmi ceux-ci, il y a les éléments les plus immédiats, les plus palpables, telles que les habitudes culinaires - élément le plus souvent pris en compte dans les écoles et qui a donné naissance à la pédagogie du couscous -, les musiques, les films, les arts, les architectures, les modes vestimentaires, les découvertes scientifiques, etc. Puis il y a une autre catégorie d'éléments beaucoup moins visibles, plus abstraits, tels que les croyances, les représentations du politique, les modèles de références, les maladies, les guerres, les paix, les risques liés aux pollutions, les valeurs sociétales (la démocratie, les droits humains, la justice), etc.

Ce processus de prise de conscience devrait néanmoins tenir compte du fait que la représentation du voyage missionnaire demeure profondément ancrée en chacune et chacun de nous. Nier cela reviendrait peut-être à renforcer le sentiment de crainte vis-à-vis de l'altérité. Avoir l'honnêteté de reconnaître que nous sommes encore habités par l'idée que l'Autre représente un danger potentiel permet d'y faire face et nous mène au second axe de réflexion.

Celui-ci devrait permettre de travailler sur ce que cette crainte et cette méfiance - substrats du voyage missionnaire - ont engendré comme misère : massacres des Amérindiens et des Aborigènes d'Australie, l'esclavage, le colonialisme, la Shoah, les conflits ethniques, la ségrégation, etc. Une prise de conscience - ou une avancée morale selon Jacques Derrida - a pris naissance en Occident depuis la fin de la Seconde Guerre mondiale, sur la nécessité de valoriser le respect des différences. Elle s'est exprimée plus ou moins symétriquement sur trois plans.

Premièrement, en particulier à travers les écrits d'anthropologues, les idées se sont mues vers le respect de la diversité des cultures et des peuples:

La tolérance n'est pas une position contemplative, dispensant les indulgences à ce qui fut ou à ce qui est. C'est une attitude dynamique, qui consiste à prévoir, à comprendre et à promouvoir ce qui veut être. La diversité des cultures humaines est derrière nous, autour de nous et devant nous. La seule exigence que nous puissions faire valoir à son endroit (créatrice pour chaque individu des devoirs correspondants) est qu'elle se réalise sous des formes dont chacune soit une contribution à la plus grande générosité des autres. (Lévi-Strauss, 1952/2001, p. 120).

Les premiers engagements de l'Unesco dans la lutte contre les préjugés raciaux dès 1949, les Déclarations des Droits humains qui ne cessent de s'amplifier et de se diffuser, le travail de mémoire autour de la Shoah et le lent processus de reconnaissance du génocide arménien, sont autant de signes qui peuvent être interprétés comme un progrès moral.

Deuxièmement, des mouvements de libération et de valorisation des minorités et des différences jusque-là discriminées se mettent en branle, réclament - et parfois obtiennent - des droits et des reconnaissances. Le droit des peuples à disposer d'eux-mêmes est proclamé, le lent processus de décolonisation s'enclenche, les Gender Studies, les Black Studies, les Folk Culture et autres Subculture Stu- 
dies émergent au sein des milieux universitaires et la discrimination positive progresse, même si là aussi il y a le danger de tomber dans d'autres extrêmes, tel le multiculturalisme.

Associé à ces deux pans, le troisième pan de l'évolution morale consiste à effectuer le passage d'un paradigme assimilationniste à celui de l'intégration, voire interculturel. Peu à peu, le nord de l'Europe s'est rendu compte que les ouvrières et ouvriers migrants étaient des personnes : "Nous voulions des bras et ce sont des être humains qui sont arrivés» (Max Frisch). La pédagogie spécifique aux étrangers - qui tend à stigmatiser et exclure - a lentement laissé la place à une pédagogie interculturelle ouverte sur la diversité et respectueuse de celle-ci, du moins dans les discours. Afin de réaliser ses objectifs, l'école devrait permettre la reconnaissance mutuelle et effective entre tous, offrir les outils qui permettent d'aller vers l'Autre sans crainte et d'ouvrir enfin au dialogue plutôt que de perpétuer le monologue des détenteurs de la violence symbolique, comme dirait Bourdieu. Pour ce faire - et comme nous nous sommes efforcés de l'argumenter jusqu'ici -, l'interculturalité ne peut se réduire aux minorités visibilisées et problématisées, comme le sont en général depuis quelques années les enfants des Balkans, les enfants d'Afrique ou les enfants porteurs d'handicaps.

Le défi de la pensée migrante, par la médiation de l'école, pourrait être la transmission du projet de Frantz Fanon, cité par Gaston Kelman, où chaque personne aurait "[...] à s'écarter des voies inhumaines qui furent celles de leurs ancêtres respectifs afin que naisse une authentique communication» (2005, p. 49). Kelman explicite en ces termes la pensée de Fanon : «Il voudrait être tout simplement un homme, et cette revendication est la colonne vertébrale de son essai, parce qu'il sait que Noir et Blanc, Juif et Aryen, sont autant de créations hybrides qui ne prennent un sens réel que quand l'homme cesse d'être un homme pour devenir un loup, un prédateur, mû par de bas instincts de la recherche d'une survie égoïste et barbare.» (Kelman, 2005, p. 50)

L'école, pour reprendre Abdallah-Pretceille, devrait être le lieu de formation de la conscience citoyenne où se transmettent les valeurs partagées pour construire un monde où la violence ne serait pas l'unique médium entre les humains. Pourtant, si la recherche en sciences de l'éducation n'appuie pas une telle ambition, celleci risque de n'être, au mieux, qu'un voeu pieux et au pire, un slogan donnant bonne conscience aux divers "missionnaires modernes». En effet, il ne suffit pas de proclamer une éducation interculturelle ouverte à tous les élèves et comprenant toutes les disciplines. Pour que celle-ci advienne, une réflexion sur le contenu et les méthodes d'enseignement de chaque discipline ne peut être évitée. Comment faire pour qu'enfin l'enseignement des langues, par exemple, ne soit plus vécu comme une corvée, un apprentissage fastidieux, mais comme une passerelle vers la diversité, une ouverture sur un autre univers? Comment s'y prendre pour que l'enseignement du français, de l'histoire ou de la géographie ait vé- 
ritablement un contenu interculturel? Voilà les enjeux auxquels la recherche en interculturalité doit impérativement répondre.

\section{Conclusion}

Nous avons essayé de mettre en lumière la nécessité, voire l'urgence pour notre société, d'introduire et de pérenniser la pensée migrante dans l'institution scolaire, à tous les niveaux. Bien entendu, les cours d'interculturalité au sein des Hautes écoles pédagogiques ont été souhaités dans la formation de toutes et tous les enseignant-e-s de l'école publique par la CDIP en 2000, mais qu'en est-il concrètement ? Le nombre d'heures enseignées se restreint d'année en année et les conditions d'enseignement se dégradent au fil du temps. Comment expliquer ce phénomène alors que nous n'avons jamais autant parlé d'intégration et de citoyenneté ? Peut-être une réponse valable vient-elle de Jacques Demorgon:

Trop souvent, les idéologies, voire les politiques, prétendent détenir des solutions [...]. Ils proposent celles-ci au vote des citoyens comme si l'exercice du vote était performatif et comportait automatiquement la réalisation des choix dominants effectués. Malheureusement, il n'en est rien et l'on voit bien où nous en sommes. L'absence d'horizon qu'il faudrait réellement atteindre par ses propres actions crée une anxiété permanente dans les populations. Elle les fait se détourner, se désintéresser de la cause publique comme la faiblesse des participations aux élections le démontre bien (Demorgon, 2005, p. 204).

Les approches interculturelles au sein de l'enseignement obligatoire se limitent, encore trop souvent, aux élèves migrants a priori porteurs d'handicaps culturels et/ou linguistiques. Ainsi, ces approches, lorsqu'elles sont appliquées en classe, sont fréquemment teintées d'un sentiment de charité envers les élèves issus de la migration, ce qui ne fait qu'augmenter et renforcer la distance entre ceux qui donnent et ceux qui reçoivent, partant du présupposé que les premiers sont les autochtones et les seconds les migrants. Dans ce cas, l'interculturalité est conçue comme une réponse "généreuse» à une situation négative.

Pourtant, cette manière de percevoir les approches interculturelles et leurs apports n'est pas la seule possible. Par exemple, dans les programmes des Hautes études de commerce, il est entendu depuis longtemps que l'interculturalité est une nécessité pour accroître le marché en touchant des publics cibles de manière culturellement adéquate. Dans la même veine, les grands entreprises multinationales offrent des cours d'interculturalité pour leurs cadres et font ainsi le beurre de bien des entreprises de communication.

À partir de ces constats, il semblerait que lorsqu'il y a un gain économique direct, l'interculture soit intéressante et potentiellement moteur de mobilisation, de financements et de perceptions positives à son égard. Mais doit-on simplement accepter cela comme un état de fait irréversible ou pouvons-nous avoir 
d'autres ambitions - plus humanistes et généreuses - envers l'interculture et son insertion dans l'école obligatoire?

Nous faisons le pari - qui nous apparaît comme une nécessité vitale - que la deuxième option est possible et c'est pourquoi nous avons tenté d'exposer une acception large de l'interculture, qui dépasse les ethnocentrismes obsolètes paralysants.

Face aux multiples situations d'interculture qui se présentent au quotidien, l'école a le devoir de préparer chaque élève à ce qu'il puisse les percevoir comme autant de chances qui lui sont données pour expérimenter, découvrir et s'ouvrir. Nous pouvons espérer que l'enfant d'aujourd'hui - l'adulte du monde de demain - habité et consolidé par la pensée migrante, sera en mesure de vivre avec sérénité les situations où l'altérité, quelle qu'elle soit, loin d'être une menace ou un danger pour son identité, sera vécue comme une opportunité.

L'éducation interculturelle, pour être prise au sérieux et lui permettre de déployer ses innombrables potentialités, doit dépasser, et de loin, la problématique des enfants étrangers dans les classes d'école. Il en va de notre responsabilité face aux adultes de demain.

\section{Notes}

1 Extraits du site internet du Fonds national suisse, «Résultats finaux du Programme national de recherche PNR 39», consultés le 8 mai 2007. http://www.snf.ch/F/NewsPool/Seiten/mm_03jan27.aspx

2 Idem.

3 Cilette Cretton, Lettre d'information DGEO $n^{\circ} 6$ du 21 février 2005.

4 Lettre d'information DGEO $n^{\circ} 3$ du mois de novembre 2004.

5 Chaque institution étatique remplit bien entendu un rôle spécifique, avec des devoirs distincts assignés par l'État. Pourtant, il arrive que les devoirs des unes entrent en contradiction avec ceux des autres. Cela est le cas dans le Canton de Vaud par rapport aux enfants des familles sans permis de séjour valide. L'école a le devoir d'intégrer ces enfants au nom des Droits de l'homme et la police, dont le devoir est le contrôle et la surveillance, a le devoir de mettre à jour et d'expulser les personnes illégales sur le territoire suisse.

6 À ce sujet, voir par exemple les ouvrages de Jacques Demorgon: L'exploration interculturelle: pour une pédagogie internationale (1989/1991); Complexité des cultures et de l'interculturel (1996/2000); L'histoire interculturelle des sociétés (1998/2002); Guide de l'interculturel en formation (1999).

7 Accord trouvé sur le site officiel de l'Office franco-allemand pour la jeunesse, consulté le 17 avril 2007. [www.ofaj.org/page.php?nav=commun/scripts/pages_dyn.php?page=accord.htm\&lng=fr]

8 La distinction entre globalisation et mondialisation est développée par le philosophe italien Giacomo Marramao dans le premier chapitre de Passaggio a Occidente (2003).

9 Grin F. : "De plus en plus, les tendances uniformisantes - qui sont à l'œuvre dans la dynamique des langues - privilégient une langue : l'anglais». 


\section{Références bibliographiques}

Abdallah-Pretceille, M. (1986). Vers une pédagogie interculturelle. Paris: Presses de la Sorbonne.

Allemann-Ghionda, C., de Goumoëns, C. \& Perregaux, C. (1999). Pluralité linguistique et culturelle dans la formation des enseignants. Fribourg: Academic Press.

Ariès, P. (1998). Les fils du McDo: La McDonaldisation du monde. Paris: Harmattan

Batori, F. (2005). Rapport d'évaluation du projet «Bus plurilingue», p. 9. non-publié

Bianconi, S. (2005). "Langues, culture et politique en Suisse. Lecture désabusée d'un cas exemplaire». Langues et cultures. Le discours sur les langues en Suisse : d'un modèle d'exemple à un cas problématique? (pp. 77-83). Colloque du 11 novembre 2005, Bienne. Berne : Académie suisse des sciences humaines et sociales.

Berthoud, G., Ischy, F. \& Simonis, O. (2002). La société de l'information: la nouvelle frontière? Université de Lausanne, Institut d'anthropologie et de sociologie.

Demorgon, J. (1989). L'explorations interculturelle: pour une pédagogie internationale. Paris: A. Colin

Demorgon, J. (1998/2002). L'histoire interculturelle des sociétés. Paris: Anthropos.

Demorgon, J. \& Lipianski, E. M. [sous la dir.] (1999). Guide de l'interculturel en formation. Paris: Retz.

Demorgon, J. (2000). Linterculturation du monde. Paris: Anthropos.

Demorgon, J. (2000). Complexité des cultures et de l'interculturel; [suivi de] contre la pensée unique. Paris: Anthroopos

Demorgon, J. (2005). Critique de l'interculturel. Paris: Anthropos.

Etounga-Manguelle, D. (1991). L'Afrique a-t-elle besoin d'un programme d'ajustement culturel? Paris: Nouvelles du Sud.

Fanon, F. (1971, [1952]). Peau noire, masques blancs. Paris : Seuils, Points Essais.

Finkielkraut A. (1987). La défaite de la pensée. Paris: Gallimard folio essais

Kelman, G. (2005). Au-delà du Noir et du Blanc. Paris : Max Milo.

Leclerc, G. (2000). La mondialisation culturelle. Les civilisations à l'épreuve. Paris: Presses Universitaires de France.

Lévi-Stauss, C. (1952/2001). Race et Histoire. Race et Culture. Paris: Albin Michel/Editions UNESCO.

Maffesoli, M. (2003). Le Voyage ou la conquête des mondes (p. 6). Paris: Editions Deervy

Marramao, G. (2003). Passaggio a Occidente. Torino: Bollati Boringhieri.

Wicker, H.-R., Fibbi, R. \& Haug, W. (2003). Les migrations et la Suisse. Zurich: Seismo.

Widmer, J. 2005. «Constitution d'une Suisse plurilingue». In La diversité des langues en Suisse dans le débat public. Une analyse socio-historique des transformations de l'ordre constitutionnel des langues de 1848 à 2000 (pp. 1-30, 2e édition). Berne : Peter Lang.

Windisch, U. (1992). Les relations quotidiennes entre Romands et Suisses allemands. Lausanne: Payot.

Mots clés: interculture, citoyenneté, migratio, migration, pensée migrante, voyage 


\section{Auf dem Weg zu einem erweiterten Migrationsgedanken}

\section{Zusammenfassung}

Dieser Artikel versucht, über die sowohl in der Theorie als auch insbesondere in der Praxis häufig verbreitete reduzierte Vorstellung von Interkulturalität als einfaches Werkzeug zur Steuerung der Zuwanderung hinauszugehen.

Anstatt dialektisch über die Verbindung zwischen Migration und Interkulturalität zu streiten, verstärken wir diese, indem wir einen erweiterten Migrationsgedanken vorschlagen. Dieser umfasst eine Bewusstseinsbildung für die pan-migratio: Das bedeutet, dass wir in einer Welt immer wiederkehrender Reisen, nicht nur der Menschen, sondern auch von Waren, Ideen, Gedanken und Vorstellungen leben. Somit lässt sich die migratio nicht auf eine rein wirtschaftliche Migrationen reduzieren. Diese grundlegende Dimension unserer Zeit ernst zu nehmen bedeutet auch, die verschiedenen Ebenen der Interkulturalität, in der wir leben, ernst zu nehmen: Den konstanten intra-schweizer Dialog zwischen verschiedenen Kulturen und Sprachen, die Frage der innereuropäischen Aussöhnung und vor allem die Verbreitung der Interkulturalität in der Welt.

Schlagworte: Interkulturalität, Migration, Cultural Citizenship, pensée migrante,

\section{Per un pensiero migrante}

\section{Riassunto}

Questo articolo aspira a superare una concezione riduttiva dell'interculturalità in quanto semplice strumento della gestione dell'immigrazione così come è spesso teorizzata e soprattutto praticata. Invece di contestare il legame tra migrazione e intercultura, l'articolo cerca di rinforzarlo dialetticamente, proponendo un pensiero migrante che consiste in una presa di coscienza della pan-migratio: ossia del fatto che viviamo in un mondo di viaggio perpetuo, che non riguarda unicamente le persone ma anche le merci, le idee, i pensieri e le rappresentazioni. Così la migratio non si limita alle migrazioni economiche. Considerare seriamente questa dimensione costitutiva della nostra cultura significa considerare seriamente i differenti livelli di intercultura che noi viviamo: la costanza dialogica intrasvizzera tra le culture e le lingue differenti, la questione della riconciliazione intraeuropea e soprattutto l'interculturazione del mondo.

Parole chiave: intercultura, cittadinanza, migratio, migrazione, pensiero migrante, viaggio 


\section{Toward a migrating thought}

\section{Summary}

This article attempts to go beyond the reductionist conception of interculturality, which is often defined into theories and into practices as a simple tool to manage immigration. Instead of dialectically challenging the link between migration and interculture, we reinforce this link by suggesting the notion of migrating thought. This notion refers to the pan-migratio awareness, i.e. to the fact that we live into a world of perpetual journeys not only of persons, but also of goods, ideas, thoughts, and representations. In this perspective, the migratio phenomena can not be reduced to economical migrations. If we seriously want to consider this phenomena as a component of our time, that means we seriously have to consider the different intercultural levels we experience : the constant intra-swiss dialogue between different cultures and languages, the intra-european conciliation question, and, above all, the interculturation of the world.

Keywords: interculturality, Cultural Citizenship, migration, migrating thought. 